\title{
NOUVELLE
}

\section{Échappement thérapeutique du mélanome : la piste biomécanique}

Margaux Lecacheur, Christophe A. Girard, Marcel Deckert, Sophie Tartare-Deckert

Université Côte d’Azur, Inserm, Centre méditerranéen de médecine moléculaire (C3M), équipe

« microenvironnement, signalisation et cancer » labellisée Ligue contre le cancer 2016, bâtiment universitaire ARCHIMED, 151 route Saint-Antoine de Ginestière, 06204 Nice Cedex 03, France.

margaux.lecacheur@univ-cotedazur.fr christophe.girard@univ-cotedazur.fr marcel.deckert@univ-cotedazur.fr sophie.tartare-deckert@univ-cotedazur.fr
Thérapies ciblées et mécanismes de résistance

Le mélanome est un cancer de la peau agressif dont l'incidence ne cesse d'augmenter : on a estimé à 157400 le nombre de nouveaux cas et à 20000 le nombre de décès pour l'année 2018 en Europe. Son pronostic est mauvais aux stades avancés et métastatiques. Le mélanome est issu de la transformation maligne d'un mélanocyte, cellule responsable de la pigmentation. L'agressivité du mélanome est principalement due à une importante hétérogénéité intra-tumorale associée à une résistance aux traitements et à un potentiel élevé de dissémination. La majorité des mélanomes cutanés résulte de mutations oncogéniques qui conduisent à l'activation constitutive de la voie MAPK (mitogen-activated protein kinases) ERK (extracellular signal regulated kinase). On retrouve des mutations de BRAF (B-rapidly accelerated fibrosarcoma) dans 40 à $50 \%$ des cas, NRAS (neuroblastoma ras viral oncogene) dans 20 à $30 \%$ des cas, ou NFl (neurofibromatosis type 1) dans 10 à $15 \%$ des cas (Figure 1) [1]. La mutation substitutive BRAF $^{V 600}$ a conduit au développement d'inhibiteurs sélectifs (BRAFi : vémurafénib ou dabrafénib), utilisés en clinique en combinaison avec un inhibiteur de la kinase MEK (MAPK/ERK kinase) (MEKi : cobimétinib ou tramétinib) pour le traitement des mélanomes non résécables chirurgicalement ou métastatiques. Pour les patients non porteurs de la mutation de BRAF ou ceux ayant rechuté après les bithérapies ciblées, d'autres stratégies thérapeutiques existent, comme les immunothérapies visant à réactiver les réponses immunitaires contre les cellules tumorales. Malgré les taux élevés (environ $75 \%$ ) de réponse à ces traitements, certains patients n'y répondent pas, et beaucoup échappent à ces thérapies à cause de l'émergence de résistances des cellules tumorales. Plusieurs mécanismes de résistance primaire, adaptative et secondaire ont été décrits. Le plus souvent, les résistances secondaires ont une origine génétique et peuvent conduire à la réactivation de la voie MAPK ERK, par des mutations de NRAS notamment. Dans d'autres cas, la résistance acquise a une origine non-génétique, caractérisée par un remodelage du paysage transcriptionnel avec la surexpression de récepteurs à activité tyrosine kinase comme AXL ou PDGFR (platelet-derived growth factor receptor), l'activation de voies de survie alternatives et la transition vers un phénotype cellulaire mésenchymateux [2]. Malgré les progrès de la prise en charge clinique du mélanome métastatique au cours des dix dernières années, les réponses obtenues demeurent insuffisantes et les patients échappent quasisystématiquement aux traitements. Ce constat nous oblige à repenser l'utilisation des thérapies ciblées et à envisager des alternatives thérapeutiques prenant en compte notamment une vision intégrée de la cellule de mélanome incluant son microenvironnement.
Plasticité des cellules de mélanome et changements phénotypiques

Les cellules de mélanome font preuve d'une remarquable plasticité créant une hétérogénéité intra-tumorale importante. Les mélanomes sont constitués de plusieurs sous-populations cellulaires, dont deux majeures: la population des cellules «prolifératives» et celle des cellules «invasives», qui possèdent des signatures transcriptionnelles distinctes [2, 3]. L'état prolifératif est principalement contrôlé par le facteur de transcription MITF (microphthalmia-associated transcription factor), un déterminant essentiel du lignage mélanocytaire. Les cellules qui expriment MITF (MITFigh) sont principalement prolifératives, expriment des marqueurs de différenciation, et sont dépourvues de capacité invasive. À l'inverse, les cellules MITFlow sont dédifférenciées, peu prolifératives, mais particulièrement invasives, et elles expriment des marqueurs mésenchymateux comme ZEBl ou la fibronectine, ou des marqueurs de résistance comme AXL. Récemment, notre équipe a montré que cette sous-population de cellules exprime également PTX3 (pentraxin 3), une protéine de l'inflammation contrôlant la voie NFKB [4]. Ainsi, les cellules prolifératives peuvent évoluer vers un phénotype invasif et vice-versa. Ce processus dynamique de changement phénotypique fournit ainsi des capacités de survie et d'adaptation aux cellules de mélanome au cours du développement 


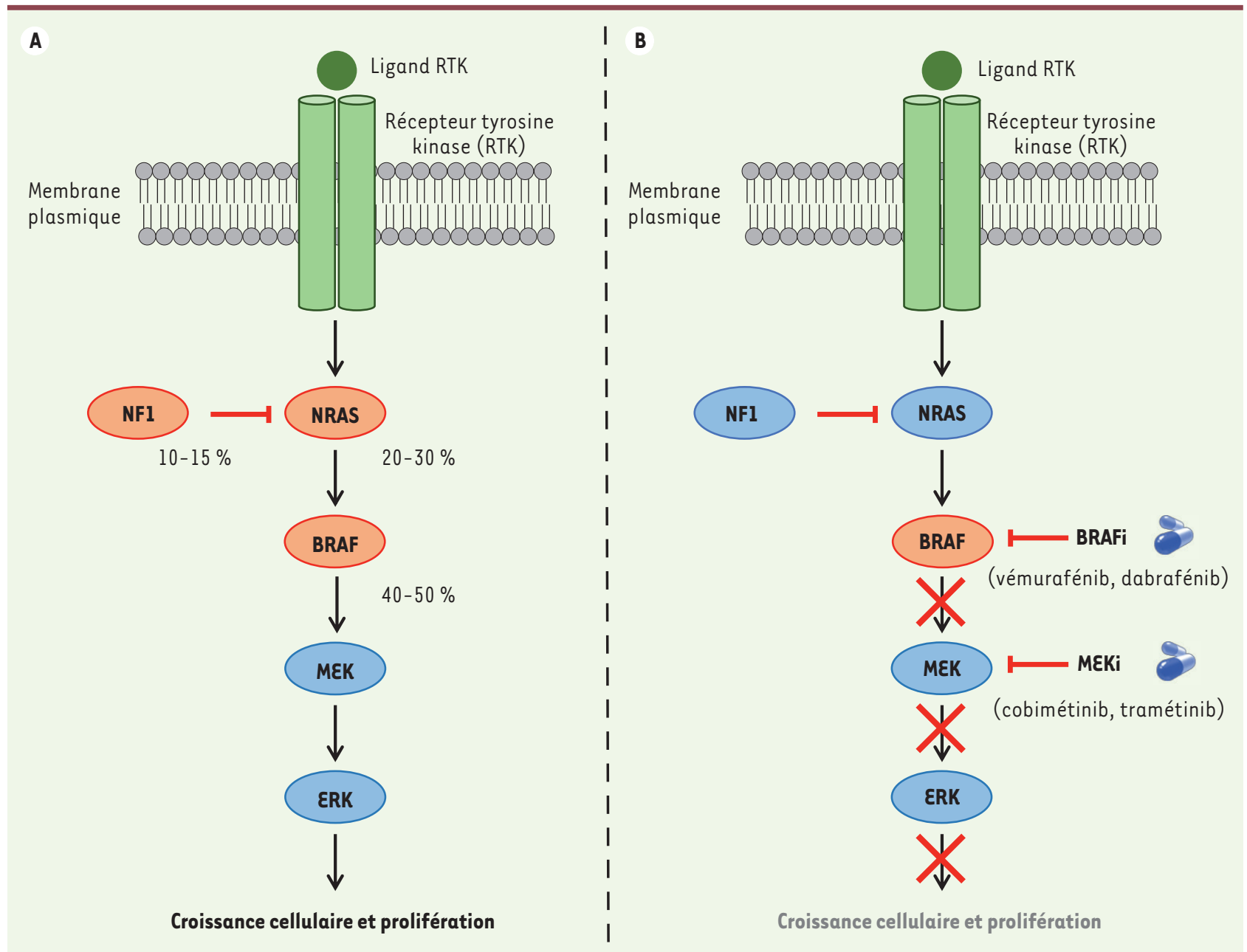

Figure 1. Principales altérations de la voie de signalisation des MAP kinases dans le mélanome et thérapies associées. A. Plus de $80 \%$ des mélanomes présentent des mutations d'un des composants de la voie de signalisation des MAP kinases. Les mutations de BRAF (V600E ou V600K) sont les plus courantes, suivies des mutations oncogéniques de NRAS, et des pertes de fonction de NF1 (une protéine inhibitrice de RAS). Ces mutations conduisent à l'activation constitutive de la voie des MAP kinases et à la prolifération incontrôlée des mélanomes. NFl, neurofibromatosis type 1 ; NRAS, neuroblastoma ras viral oncogene; BRAF, B-rapidly accelerated fibrosarcoma; MEK, MAPK/ERK kinase ; ERK, extracellular signal-regulated kinase. B. Thérapies des mélanomes avec mutation de BRAF (V600E ou V600K). Ces mélanomes sont actuellement traités avec une combinaison d'inhibiteurs ciblant la kinase BRAF et son principal effecteur, MEK. Ces thérapies ciblées inhibent la voie des MAP kinases et stoppent la prolifération des cellules de mélanome. BRAFi, inhibiteur de BRAF ; MEKi, inhibiteur de MEK.

tumoral. La tendance à transiter vers un phénotype mésenchymateux est favorisée par des signaux provenant du microenvironnement tumoral comme l'inflammation, la carence en oxygène ou en nutriments, mais est aussi causée par les thérapies ciblées ellesmêmes. Dans ce contexte, l'acquisition d'une signature mésenchymateuse de type MITFlow/AXL high représente un mécanisme majeur de résistance nongénétique aux thérapies ciblées, qui est responsable des rechutes [5].
Microenvironnement matriciel et mécanotransduction

Le mélanome se développe dans un environnement riche en cellules non cancéreuses, dont le fonctionnement est altéré par les cellules cancéreuses [6] $(\rightarrow)$. On y retrouve

$\rightarrow$ Voir la Synthèse de $\varepsilon$. Buache, M.C. Rio, $\mathrm{m} / \mathrm{s} \mathrm{n}^{\circ} 4$, avril 2014, page 385 des cellules endothéliales, des cellules immunitaires et des fibroblastes activés en myofibroblastes (cancer-associated fibroblasts, CAF), dont le princi- pal rôle est de synthétiser et remodeler la matrice extracellulaire. En plus des facteurs intrinsèques liés à des altérations génétiques et épigénétiques de la cellule tumorale, des facteurs extrinsèques provenant du stroma et de la matrice extracellulaire influencent la réponse des mélanomes aux traitements anti-cancéreux [7]. La matrice extracellulaire est composée de molécules de structure (collagène et élastine) et d'adhérence (fibronectine), et constitue un réseau dynamique 
dont les propriétés physico-chimiques influencent l'architecture des tissus. Les tumeurs se caractérisent par un dépôt anormal de matrice extracellulaire. L'augmentation du dépôt et de l'organisation des fibres de collagène conduit à une rigidité accrue de la matrice extracellulaire tumorale par rapport à celle des tissus normaux. Les cellules tumorales sont capables de percevoir ces forces mécaniques et d'y répondre grâce à différents récepteurs de surface, tels que les intégrines [8]. Ce processus de mécanotransduction convertit un signal mécanique en un signal biochimique. Les intégrines sont les récepteurs de la fibronectine et du collagène les mieux décrits. La voie de signalisation des intégrines est suractivée dans de nombreux cancers, et participe à des étapes clés de la progression tumorale ainsi qu'à la résistance thérapeutique. Les signaux propagés via le réseau d'acto-myosine activent la translocation nucléaire de co-activateurs de la transcription, tels que YAP (yes-associated protein) ou MRTF (myocardin-related transcription factor). YAP est le principal «mécanosenseur » des cellules tumorales [9]. II contrôle leur prolifération et la résistance aux traitements. Comme YAP, MRTF, en réponse à une augmentation de rigidité de la matrice extracellulaire, est transloqué dans le noyau, où il active l'expression de ses gènes cibles, impliqués dans la contraction du cytosquelette [10]. Comme les CAF, les cellules de mélanome ont la capacité de sécréter leur propre matrice extracellulaire, en particulier après la transition phénotypique mésenchymateuse qui a lieu en réponse aux traitements ciblés [11].

La résistance mésenchymateuse aux thérapies ciblées confère à la cellule de mélanome des propriétés de fibroblastes activés Nos recherches sur les processus de résistance non-génétique associée à la population MITFlow/AXL high nous ont conduits à identifier un phénotype remarquable de ces cellules lié à la plasticité mécanique [12]. Par des approches multidisciplinaires fondées sur des modèles de culture cellulaire sur matrices synthétiques de collagène de rigidité contrôlée et sur des techniques d'imagerie optique et biophysique de la matrice extracellulaire, nous avons montré que la résistance non-génétique associée à l'acquisition d'un phénotype mésenchymateux s'accompagne d'une forte sensibilité aux contraintes mécaniques de la matrice extracellulaire. En analysant la signalisation de la mécanotransduction, nous avons observé que les

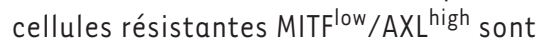
plus sensibles à la rigidité matricielle que les lignées parentales sensibles aux BRAFi. Leur ensemencement sur un substrat rigide stimule leur prolifération et s'accompagne d'un étalement plus prononcé, de la formation d'un grand nombre d'adhérences focales, d'un remodelage du cytosquelette d'actine et de l'activation des mécanosenseurs YAP et MRTF (Figure 2). En revanche, cette «mécanosensibilité » n'est pas observée dans les mélanomes ayant acquis une résistance génétique par une mutation de NRAS. Les cellules résistantes mésenchymateuses possèdent d'autres propriétés des CAF et des myofibroblastes présents dans les fibroses [11]. Elles produisent des forces de tension et de remodelage du collagène, et synthétisent une matrice extracellulaire abondante et fibrillaire, qui protège les mélanomes porteurs de mutations de BRAF «naïfs» des thérapies ciblées. Enfin, le ciblage de YAP ou MRTF inhibe les propriétés contractiles des cellules MITFlow $/ \mathrm{AXL}^{\text {high }}$ et restaure leur sensibilité thérapeutique, démontrant l'implication des voies de la mécanotransduction dans la résistance aux thérapies ciblées. Des analyses bioinformatiques des biopsies de mélanomes humains indiquent également que l'expression de gènes liés à la mécanotransduction et au remodelage matriciel est associée à l'échappement aux thérapies ciblées et aux rechutes [12].

\section{Les thérapies ciblées induisent} une reprogrammation biomécanique des mélanomes avec mutation de BRAF Nous avons ensuite cherché à savoir si cette reprogrammation mécanique résultait d'une adaptation rapide des cellules sensibles aux inhibiteurs de la voie MAP ERK. Nous avons donc traité plusieurs lignées sensibles par Vémurafénib ou Tramétinib. In vitro, I'inhibition de la voie MAP ERK induit un changement morphologique et un remodelage du cytosquelette d'actine, accompagné de la translocation nucléaire de YAP et MRTF et de l'expression accrue de plusieurs de leurs gènes cibles (Figure 2). De plus, ce traitement augmente la contraction des cellules sensibles, et conduit au dépôt d'une matrice extracellulaire remodelée capable de conférer une résistance thérapeutique à des mélanomes «naïfs». Nous avons ensuite confirmé dans des modèles de xénogreffe de mélanome humain avec mutation de BRAF issus de lignées cellulaires ou dérivés de biopsies de patient (patient-derived xenograft, $P D X)$, que les thérapies ciblées provoquent un remodelage du stroma tumoral, un alignement des fibres de collagène et une rigidification de la tumeur. Par ailleurs, I'inhibition pharmacologique de YAP prévient le remodelage matriciel induit par les thérapies ciblées et améliore leur efficacité [12].

\section{Perspectives}

Nos travaux ont révélé un nouveau mécanisme d'adaptation des mélanomes avec mutation de la protéine BRAF aux thérapies ciblées, qui conduit à la production et au remodelage de la matrice extracellulaire, à la rigidification de la tumeur et à l'activation de la signalisation de la mécanotransduction. L'inhibition de la voie MAPK alimente une boucle mécanique 


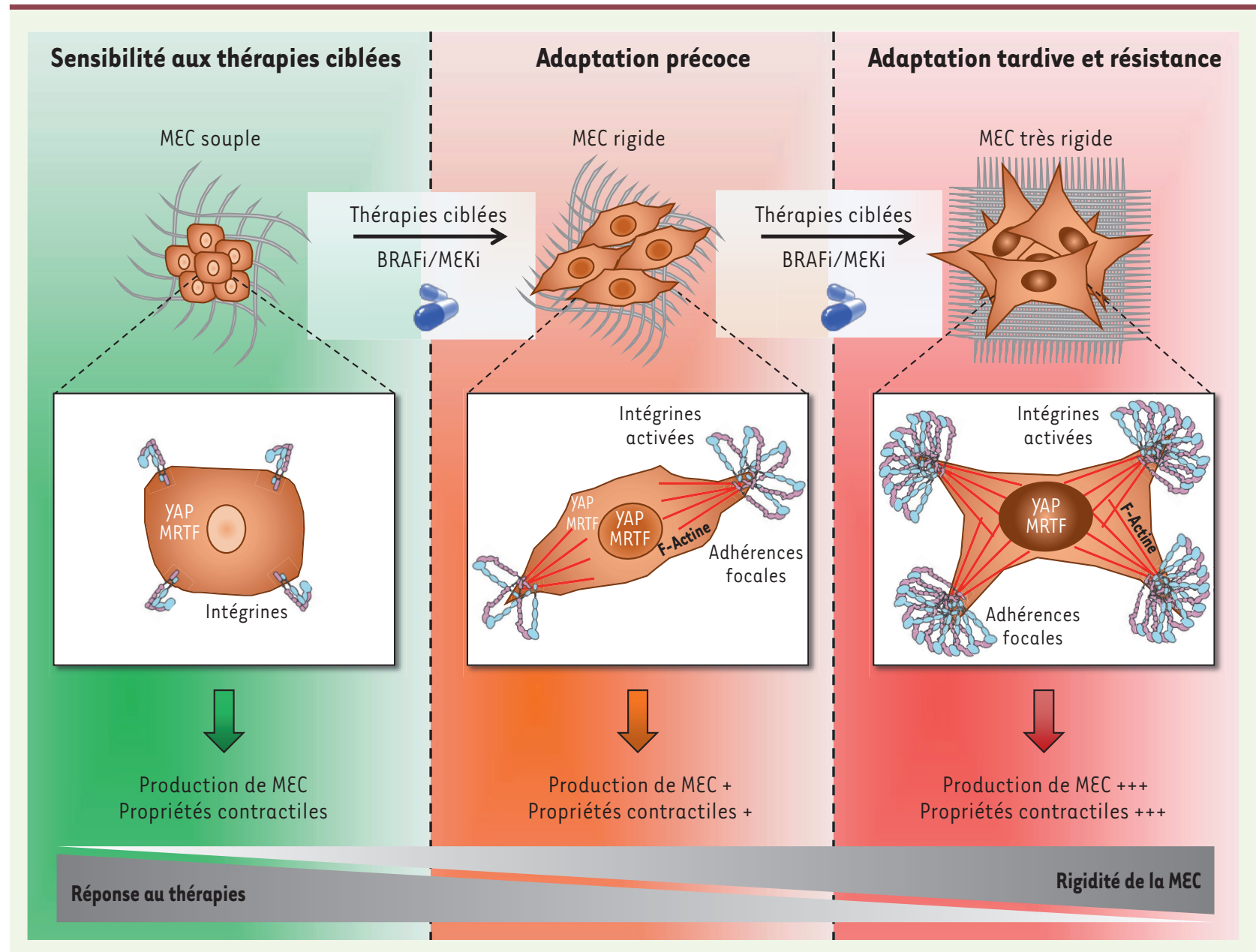

Figure 2. Les thérapies ciblant la voie oncogénique $B R A F^{V 600 E / K}$ induisent une reprogrammation biomécanique des cellules de mélanome. Le traitement combiné par des inhibiteurs de BRAF (BRAFi) et de MEK (MEKi) promeut une rigidification de la tumeur par une boucle de rétrocontrôle positif entre les signaux biomécaniques transmis par la matrice extracellulaire (MEC) et les voies intracellulaires de la mécanotransduction, conduisant à un échappement thérapeutique. En réponse aux thérapies ciblées, les cellules de mélanome s'adaptent en produisant une MEC abondante et fibrillaire, qui participe à la rigidification de la tumeur et à l'activation des voies de signalisation mécanosensibles. Cette boucle entraîne une activation des intégrines, avec une augmentation du nombre d'adhérences focales, et une réorganisation du cytosquelette d'actine associée à une polymérisation de l'actine en actine filamenteuse (F-actine). Ces évènements moléculaires induisent la translocation au noyau des co-activateurs de la transcription YAP (yes-associated protein) et MRTF (myocardin-related transcription factor), et l'activation de programmes transcriptionnels impliqués dans la production de forces contractiles, le remodelage de la MEC environnante, et la résistance aux thérapies ciblées. À l'inverse, une MEC « souple » n'active que faiblement les intégrines, l'assemblage des adhérences focales, et maintient YAP et MRTF dans le cytoplasme. En l'absence de signaux biomécaniques, la cellule cancéreuse est plus sensible aux thérapies ciblées.

de rétroaction positive, dans laquelle les co-activateurs de la transcription YAP et MRTF jouent un rôle pivot en favorisant la résistance aux thérapies ciblées. De plus, le ciblage de YAP prévient la réponse adaptative aux thérapies ciblées et améliore leur efficacité. Nos résultats indiquent que prévenir le dialogue biomécanique des mélanomes avec leur microenvironnement matriciel permet- trait de prolonger l'action des thérapies ciblées, d'éviter les rechutes, et d'améliorer la prise en charge des patients atteints de mélanome métastatique. $\diamond$ Melanoma therapeutic escape: the biomechanical track

\section{LIENS D'INTÉRÊT}

Les auteurs déclarent n'avoir aucun lien d'intérêt concernant les données publiées dans cet article.

\section{RÉFÉRENCES}

1. Cancer Genome Atlas N. Genomic classification of cutaneous melanoma. Cell $2015 ; 161$ : 1681-96.

2. Rambow F, Marine JC, Goding CR. Melanoma plasticity and phenotypic diversity: therapeutic barriers and opportunities. Genes Dev 2019 ; 33 : 1295-318.

3. Hoek KS, Schlegel NC, Brafford P, et al. Metastatic potential of melanomas defined by specific gene expression profiles with no BRAF signature. Pigment Cell Res 2006; $19: 290-302$. 


\section{RÉFÉRENCES}

4. Rathore M, Girard C, Ohanna M, et al. Cancer cellderived long pentraxin 3 (PTX3) promotes melanoma migration through a toll-like receptor 4 (TLR4)/

NF-kappaB signaling pathway. Oncogene 2019 ; 38 : 5873-89.

5. Hugo W, Shi H, Sun L, et al. Non-genomic and immune evolution of melanoma acquiring MAPKi resistance. Cell 2015 ; 162 : 1271-85.

6. Buache $\varepsilon$, Rio MC. Le stroma tumoral : un terreau fertile pour la cellule cancéreuse. Med Sci (Paris) $2014 ; 30: 385-90$.
7. Almeida FV, Douglass SM, Fane ME, Weeraratna AT. Bad company: Microenvironmentally mediated resistance to targeted therapy in melanoma. Pigment Cell Melanoma Res 2019; $32: 237-47$.

8. Levental KR, Yu H, Kass L, et al. Matrix crosslinking forces tumor progression by enhancing integrin signaling. Cell $2009 ; 139$ : 891-906.

9. Dupont S, Morsut L, Aragona M, et al. Role of YAP/TAZ in mechanotransduction. Nature $2011 ; 474$ : 179-83.

10. Foster CT, Gualdrini F, Treisman R. Mutual dependence of the MRTF-SRF and YAP-TEAD pathways in cancer-associated fibroblasts is indirect and mediated by cytoskeletal dynamics. Genes Dev 2017 ; $31: 2361-75$

11. Diazzi S, Tartare-Deckert S, Deckert M. Bad neighborhood: fibrotic stroma as a new player in melanoma resistance to targeted therapies. Cancers $2020 ; 12: 1364$.

12. Girard CA, Lecacheur $M$, Ben Jouira $R$, et al. A Feed-forward mechanosignaling loop confers resistance to therapies targeting the MAPK pathway in BRAF-mutant melanoma. Cancer Res $2020 ; 80: 1927-41$ 